\title{
Kaons in a hot and flavor-asymmetric medium
}

\author{
M.C. Ruivo, C.A. de Sousa, C. Providência \\ Departamento de Física, Universidade de Coimbra, P-3004-516 Coimbra, Portugal \\ Received 10 November 1998; revised 15 February 1999; accepted 17 February 1999
}

\begin{abstract}
We investigate the behavior of kaons in a flavor-asymmetric quark medium at finite temperature, within SU(3) Nambu-Jona-Lasinio type models. The effect of the Fermi sea on kaon properties is weaker in the model which includes a vector-pseudovector interaction than in the model without it. The study of the combined effect of density and temperature on the vacuum and kaon properties shows that the low temperature regime does not change significantly the effects observed at $T=0$; as the temperature increases the mass splitting between $K^{+}$and $K^{-}$modes, however reduced, is still meaningful. (C) 1999 Elsevier Science B.V. All rights reserved.
\end{abstract}

Keywords: NJL model; Chiral symmetry; Three-flavor thermodynamics; Flavor-asymmetric matter

\section{Introduction}

One of the important questions in intermediate and high energy physics is the understanding of strongly interacting matter as a function of temperature and density. It has been shown in the framework of some models that, at sufficiently high temperature and/or density, spontaneously broken symmetries are restored. Restoration of symmetry is also expected to hold for the dynamically broken chiral symmetry in QCD. It is believed that this phenomenon could occur in heavy-ion collisions, the early universe and neutron stars.

For zero density and finite temperature the most reliable existing information comes from lattice calculations, indicating that both the chiral transition and the confinementdeconfinement phase transition take place at the same temperature $T_{c} \simeq 150 \mathrm{MeV}$ [1]. For finite densities no firm lattice results are available. In this situation, simplified models may give some insight into hadronic matter at finite densities and temperatures which is necessary for the interpretation of the experimental data that have started to appear. 
The problem of how the properties of the hadrons change in hot nuclear matter in comparison to their free values is nowadays an important issue in nuclear and particle physics [2]. In particular, the study of possible modifications of pseudoscalar mesons is extremely interesting since, due to their Goldstone boson nature, they could provide a signal for the restoration of chiral symmetry. Since the pioneering work of Kaplan and Nelson [3] a great amount of theoretical work on kaons in flavor-asymmetric medium has been reported [4-11]. A conclusion emerging from most of the theoretical attempts of dealing with this problem is the prediction of a slightly raising mass for the $K^{+}$and a decreasing mass for the $K^{-}$, a prediction which is supported by the analysis of the data on kaonic atoms [12]; the way in which such splitting occurs is of course model dependent.

Fundamental changes in meson properties seem necessary to explain experimental results such as those from KaoS and FOPI collaborations at GSI [13-15]. The $K^{+}$and $K^{-}$yield are comparable at the same available energy, which is surprising since $K^{-}$ production is suppressed in $p p$ collisions by an order of magnitude at the same available energy. The elementary rate of $K^{-}$is lower and its absorption is stronger. Recent data [15] on kaon production in $\mathrm{Ni}+\mathrm{Ni}$ collisions show a $K^{-} / K^{+}$rate larger than in nucleon-nucleon collisions, which indicates an enhancement of the $K^{-}$production in the medium.

It is well known that a many-body system, such as a hadronic medium, can exhibit a great variety of particle-hole excitations that may carry quantum numbers of the hadrons under investigation, the kaons in the present case. The interplay between the different modes may produce changes in kaon properties.

The pursuit of theoretical approaches in order to understand the in-medium kaon behavior remains challenging. The Nambu-Jona-Lasinio (NJL) model [16] model is very successful in the description of many features in hadron physics. This model is also suited to study the chiral transition of infinite uniform quark system. The two-flavor NJL model has been used for this purpose by many authors [17-20]. The chiral transition for the three-flavor case as well as kaon properties have been studied at zero chemical potential [21,22] and at non-zero chemical potential [23,24,5].

Our program is to examine the properties of kaons as a function of density and temperature taking into account the Fermi sea effects.

Recently, we have studied the behavior of kaons in a type of asymmetric quark matter (simulating symmetric nuclear matter: $\rho_{l l}=\rho_{d}, \rho_{s}=0$ ) and the new findings concern mainly flavor-asymmetric effects on kaonic modes [7]. We have observed the splitting between $K^{ \pm}$and the occurrence of a low-lying $K^{-}-$like branch at low baryonic densities. This branch is not significantly affected by the inclusion of strange matter in the medium while the flavor asymmetry is preserved [8]. We remark that, while the $K^{ \pm}$are excitations of the Dirac sea, the low-lying branch, a non-trivial dynamical consequence of flavor symmetry breaking of the medium, is a collective particle-hole excitation of the Fermi sea.

It is pertinent to have a closer look into the behavior of the kaonic modes and the present investigation is addressed to two main points: (i) the influence of the vector- 
pseudovector interaction on the behavior of the kaons in the presence of Fermi sea effects; (ii) the role played by both density and thermal effects on the vacuum and kaon properties. The present study is based on the time-dependent Hartree-Fock theory in the formalism of the density matrix. This framework provides a unified description of the equation of state of hadronic matter and its excitations at zero and non-zero temperature.

Along the above lines, this paper is organized as follows. In Section 2 we give a review of the model and formalism at finite baryonic density and temperature. In Section 3 we present and discuss our numerical results. Section 4 states our concluding remarks.

\section{Modelling temperature and density effects}

We adopt a three-flavor version of the NJL model which, besides the scalar-pseudoscalar and vector-pseudovector interaction terms, includes the 't Hooft six fermion interaction that models the axial $U(1)_{A}$ symmetry breaking. The model is defined by the following Lagrangian:

$$
\begin{aligned}
L= & \bar{q}\left(i \gamma^{\mu} \partial_{\mu}-m\right) q+\frac{1}{2} g_{S} \sum_{a=0}^{8}\left[\left(\bar{q} \lambda^{a} q\right)^{2}+\left(\bar{q} i \gamma_{5} \lambda^{a} q\right)^{2}\right] \\
& -\frac{1}{2} g_{V} \sum_{a=0}^{8}\left[\left(\bar{q} \gamma_{\mu} \lambda^{a} q\right)^{2}+\left(\bar{q} \gamma_{\mu} \gamma_{5} \lambda^{a} q\right)^{2}\right] \\
& +g_{D}\left\{\operatorname{det}\left[\bar{q}_{i}\left(1+\gamma_{5}\right) q_{j}\right]+\operatorname{det}\left[\bar{q}_{i}\left(1-\gamma_{5}\right) q_{j}\right]\right\},
\end{aligned}
$$

where $q=(u, d, s)$ are the quark fields and $\lambda_{a}(0 \leqslant a \leqslant 8)$ are the $\mathrm{U}(3)$ flavor matrices. The model parameters are $m=\operatorname{diag}\left(m_{u}, m_{d}, m_{s}\right)$, the current quark mass matrix ( $m_{d}=m_{u}$ ), the coupling constants $g_{S}, g_{V}$ and $g_{D}$ and the cutoff in three-momentum space, $A$, which regularizes momentum space integrals. In order to discuss the effects of the vector-pseudovector interaction we are going to consider two models: model I with $g_{V}=0$ and model II with $g_{V} \neq 0$. The set of parameters in the two models are chosen in order to fit the values in vacuum for the pion mass, the pion decay constant, the kaon mass and the quark condensates. For model I we have $m_{\pi}=139 \mathrm{MeV}, f_{\pi}=$ $93.0 \mathrm{MeV},\langle\bar{u} u\rangle=\langle\bar{d} d\rangle=-(246.7 \mathrm{MeV})^{3},\langle\bar{s} s\rangle=-(266.9 \mathrm{MeV})^{3}, m_{K}=495.7 \mathrm{MeV}$, $f_{K}=98.9 \mathrm{MeV}$, with the parameter set $\Lambda=631.4 \mathrm{MeV}, g_{S} \Lambda^{2}=3.658, g_{D} \Lambda^{5}=-9.40$, $m_{u}=m_{d}=5.6 \mathrm{MeV}$ and $m_{s}=135.6 \mathrm{MeV}$; for model II: $m_{\pi}=140 \mathrm{MeV}, f_{\pi}=$ $93.3 \mathrm{MeV},\langle\bar{u} u\rangle=\langle\bar{d} d\rangle=-(287.0 \mathrm{MeV})^{3},\langle\bar{s} s\rangle=-(306.2 \mathrm{MeV})^{3}, m_{K}=498.7 \mathrm{MeV}$, $f_{K}=98.4 \mathrm{MeV}$, with the parameter set: $\Lambda=750 \mathrm{MeV}, g_{S} \Lambda^{2}=3.624, g_{V} \Lambda^{2}=3.842$, $g_{D} \Lambda^{5}=-9.11, m_{u}=m_{d}=3.61 \mathrm{MeV}$ and $m_{s}=88.0 \mathrm{MeV}$.

Let us discuss the equilibrium states at finite temperature and density. The groundstate of the system can be described by the density matrix given by $f=\operatorname{diag}\left(f_{u}, f_{d}, f_{s}\right)$ with

$$
f_{i}=\frac{1}{2}\left[I\left(n_{i}^{-}+n_{i}^{+}\right)-\frac{\beta M_{i}+\boldsymbol{\alpha} \cdot \boldsymbol{p}}{E_{i}}\left(n_{i}^{-}-n_{i}^{+}\right)\right] \theta\left(\Lambda^{2}-p^{2}\right),
$$


where $n_{i}^{(\mp)}(i=u, d, s)$ are the Fermi distribution functions of the negative (positive) energy states, which have the form

$$
n_{i}^{(\mp)}=\left[1+\exp \left(\mp\left(\beta\left(E_{i} \mp \Delta E_{i} \pm \mu_{i}\right)\right)\right)\right]^{-1} .
$$

In the last equation $\beta=1 / T, \mu_{i}$ is the chemical potential, $M_{i}$ is the constituent quark mass, $E_{i}=\left(p^{2}+M_{i}^{2}\right)^{1 / 2}$ and $\Delta E_{i}$ is the energy shift induced by the vector-pseudovector interaction.

To investigate the equation of state at finite density and temperature we consider the thermodynamical potential $\Omega=\mathcal{E}-T S-\sum_{i} \mu_{i} N_{i}$, where

$$
\begin{aligned}
\mathcal{E}= & -2 N_{c} V \sum_{i}\left\{\int \frac{d^{3} p}{(2 \pi)^{3}} \frac{p^{2}+m_{i} M_{i}}{E_{i}}\left(n_{i}^{-}-n_{i}^{+}\right) \theta\left(A^{2}-p^{2}\right)\right\} \\
& -g_{S} \sum_{i}\left(\left\langle\bar{q}_{i} q_{i}\right\rangle\right)^{2}+g_{V} \sum_{i}\left(\left\langle q_{i}^{+} q_{i}\right\rangle\right)^{2}-2 g_{D}\langle\bar{u} u\rangle\langle\bar{d} d\rangle\langle\bar{s} s\rangle
\end{aligned}
$$

is the internal energy,

$$
\begin{aligned}
S= & -2 N_{c} V \sum_{i} \int \frac{d^{3} p}{(2 \pi)^{3}} \theta\left(\Lambda^{2}-p^{2}\right) \\
& \times\left\{\left[n_{i}^{+} \ln n_{i}^{+}+\left(1-n_{i}^{+}\right) \ln \left(1-n_{i}^{+}\right)\right]+\left[n_{i}^{+} \rightarrow n_{i}^{-}\right]\right\}
\end{aligned}
$$

is the entropy, $V$ is the volume of the system and $N_{c}=3$.

The quark condensates and the quark densities are defined, for each one of the flavors $i=u, d, s$, respectively as

$$
\begin{aligned}
\left\langle\bar{q}_{i} q_{i}\right\rangle & =-2 N_{c} \int \frac{d^{3} p}{(2 \pi)^{3}} \frac{M_{i}}{E_{i}}\left(n_{i}^{-}-n_{i}^{+}\right) \theta\left(\Lambda^{2}-p^{2}\right), \\
\rho_{i} & =\left\langle q_{i}^{+} q_{i}\right\rangle=2 N_{c} \int \frac{d^{3} p}{(2 \pi)^{3}}\left(n_{i}^{-}+n_{i}^{+}-1\right) \theta\left(\Lambda^{2}-p^{2}\right) .
\end{aligned}
$$

The quark masses, $M_{i}$, and the energy shifts, $\Delta E_{i}$, are determined self-consistently by minimizing the thermodynamical potential $\Omega$ with respect to the constituent quark masses $M_{i}$ and to the chemical potential $\mu_{i}$, respectively. This leads to six gap equations

$$
M_{i}=m_{i}-2 g_{S}\left\langle\bar{q}_{i} q_{i}\right\rangle-2 g_{D}\left\langle\bar{q}_{j} q_{j}\right\rangle\left\langle\bar{q}_{k} q_{k}\right\rangle
$$

with $i, j, k$ cyclic, and

$$
\Delta E_{i}=2 g_{V}\left\langle q_{i}^{+} q_{i}\right\rangle
$$

The excited states are described as deformed states generated from the state of equilibrium by the application of suitable generators $S$. This means that the density matrix which describes these states is of the form $f=\exp (i S) f_{0} \exp (-i S), S$ being a hermitian time-dependent operator and $f_{0}$ the equilibrium matrix density. 
For small amplitude excitations the time evolution of the system is governed by the Lagrangian

$$
\mathcal{L}=\frac{i}{2} \operatorname{tr}\left(f_{0}[S, \dot{S}]\right)-\mathcal{E}^{(2)}(f),
$$

where $\mathcal{E}^{(2)}(f)$ is the quadratic term in the generators of the energy functional [20].

Here we choose the generator $S$ in the form

$$
S_{K}=\left(\gamma_{5} L_{1}+i \beta \gamma_{5} L_{2}+\beta \boldsymbol{\sigma} \cdot \boldsymbol{p} L_{3}+\boldsymbol{\sigma} \cdot \boldsymbol{p} L_{4}\right) \lambda_{K}+\text { c.c., }
$$

where $\lambda_{K}$ is $\left(\lambda_{4} \pm i \lambda_{5}\right) / \sqrt{2}$ for $K^{-}\left(K^{+}\right)$, and $L_{1}, L_{2}, L_{3}$ and $L_{4}$, variational functions of $p, t$, play the role of canonically conjugate coordinates. We remark that this operator is more general than the one used in our previous works [7], since now we are dealing with an anharmonic situation due to introduction of temperature. The dispersion relation derived from the Lagrangian (10) is of the form

$$
\left(1-K_{P} J_{P P}\right)\left(1-K_{A} J_{A A}\right)-K_{P} K_{A} J_{P A}^{2}=0,
$$

where $K_{P}=g_{S}+g_{D}\langle\vec{d} d\rangle$ and $K_{A}=-g_{V}$. The integrals $J_{P A}, J_{A A}$ and $J_{P P}$ obey the following relations:

$$
\begin{aligned}
& \omega J_{P A}=\left(M_{u}+M_{s}\right) J_{P P}+2\langle\bar{u} u+\bar{s} s\rangle, \\
& \omega J_{A A}=\left(M_{u}+M_{s}\right) J_{P A}+2\left\langle u^{+} u-s^{+} s\right\rangle
\end{aligned}
$$

and

$$
\begin{aligned}
J_{P P}= & 2 N_{c} \int \frac{d^{3} p}{(2 \pi)^{3}}\left\{\frac{M_{u}\left(M_{s}-M_{u}\right)-\omega E_{u}}{E_{s}^{2}-\left(\omega+E_{u}\right)^{2} E_{u}} \tanh \frac{\beta\left(E_{u}+\bar{\mu}_{u}\right)}{2}\right. \\
& \left.\times \frac{M_{u}\left(M_{s}-M_{u}\right)+\omega E_{u}}{E_{s}^{2}-\left(\omega-E_{u}\right)^{2} E_{u}} \tanh \frac{\beta\left(E_{u}-\bar{\mu}_{u}\right)}{2}+s \rightarrow u, w \rightarrow-\omega\right\}=0 .
\end{aligned}
$$

Here $\bar{\mu}_{u}=\mu_{u}-\Delta E_{u}$ and $\omega=m_{K^{+}}-\left(\Delta E_{u}-\Delta E_{s}\right)$ or $\omega=-m_{K^{-}}-\left(\Delta E_{u}-\Delta E_{s}\right)$, according to whether one wants to describe the changes in $K^{+}$or $K^{-}$mass.

In the present approach one can check that the NJL model satisfies Partial Conservation of Axial Current (PCAC) relations by deriving them consistently from the Energy Weighted Sum Rule (EWSR). For a generic mesonic state $|r\rangle$ with energy $\omega_{r}$ associated with the transition operator $\Gamma$ the strength function $F_{r}=\omega_{r}|\langle r|\Gamma| 0\rangle|^{2}$ satisfies the EWSR which reads

$$
m_{1}=\sum_{r} \omega_{r}|\langle r|\Gamma| 0\rangle|^{2}=\frac{1}{2}\left\langle\Phi_{0}|[\Gamma,[H, \Gamma]]| \Phi_{0}\right\rangle .
$$

This equation, with the transition operator defined as $\Gamma=\Gamma_{+}+\Gamma_{-}$, with $\Gamma_{ \pm}=$ $\gamma_{5}\left(\lambda_{4} \pm i \lambda_{5}\right) / \sqrt{2}$, leads to the following generalization of the PCAC relation, at finite temperature and density:

$$
\sum_{\alpha} m_{K, \alpha}^{2} f_{K, \alpha}^{2} \simeq-\left(m_{u}+m_{s}\right)[\langle\bar{u} u\rangle+\langle\bar{s} s\rangle]
$$

This relation provides us with a tool to determine the percentage of the total strength located in the kaonic modes, which indicates its degree of collectivity. 


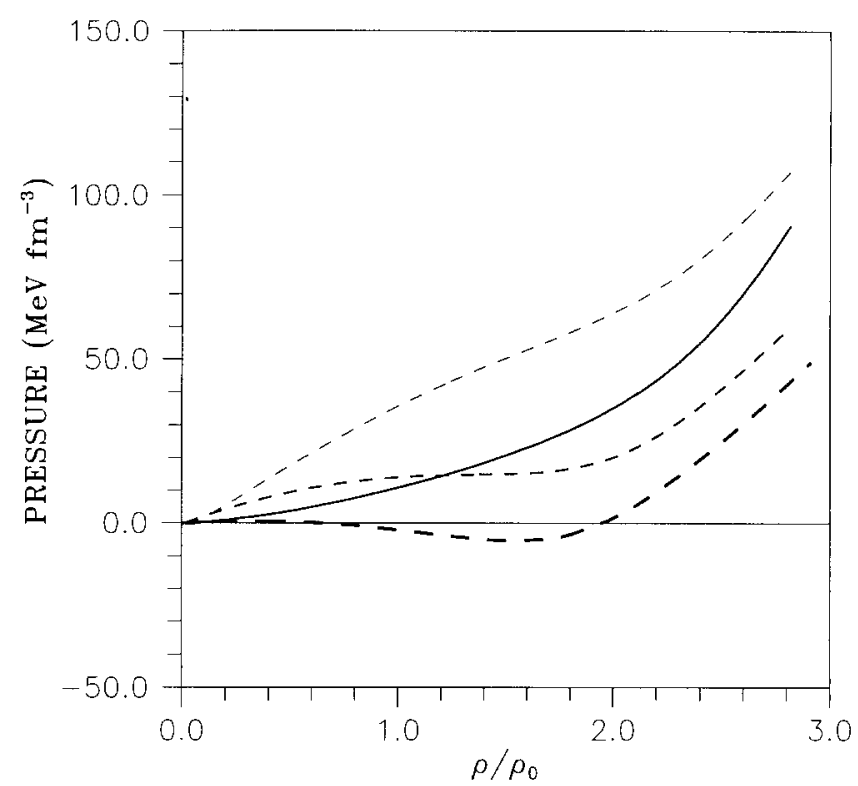

Fig. 1. Pressure versus the baryonic density for model I (dashed curves) at several temperatures: $T=0 \mathrm{MeV}$ (thick curves), $T=50 \mathrm{MeV}$ (medium-thick curves) and $T=100 \mathrm{MeV}$ (thin curves) and for model II (solid curve) at $T=0 \mathrm{MeV}$.

\section{Results}

\subsection{Comparison of the two models at finite density and zero temperature}

We start with a brief discussion on the nature of the phase transition within the two models. By performing the calculation of the vacuum properties at a fixed chemical potential as in Refs. [17-19,23] one observes that, for model I, the thermodynamical potential $\Omega$ has more than one extremum for a certain range of values of $\mu$ at low temperatures, the physical solution being the one which corresponds to the minimum of $\Omega$. Consequently, a first-order phase transition is found.

If one chooses to perform the calculation at a fixed density, as in $[25,26,7,8]$, information on the nature of the phase transition can be achieved by plotting the pressure, $P(\rho, T)=-[\Omega(\rho, T)-\Omega(\rho=0, T)]$, as a function of the density as is shown in Fig. 1. It can be seen that for model I (dashed lines) the phase transition is first order for $T \leqslant T_{c} \simeq 50 \mathrm{MeV}$. As a matter of fact for $T=0 \mathrm{MeV}$ there is a range of densities $\left(0.1 \rho_{0} \leqslant \rho \leqslant 2 \rho_{0}\right)$ where the pressure and/or the compressibility are negative, meaning that the system is in a mixed phase. As the temperature increases this range of densities becomes smaller and disappears above $T_{c}$, the pressure becoming positive and monotonously increasing with density. The conclusions concerning the phase transition diagram are equivalent to those discussed in [18] for the two-flavor NJL model.

With model II (full line of Fig. 1) the pressure and the compressibility are positive for all temperatures and the phase transition is second-order as already shown in [24]. 


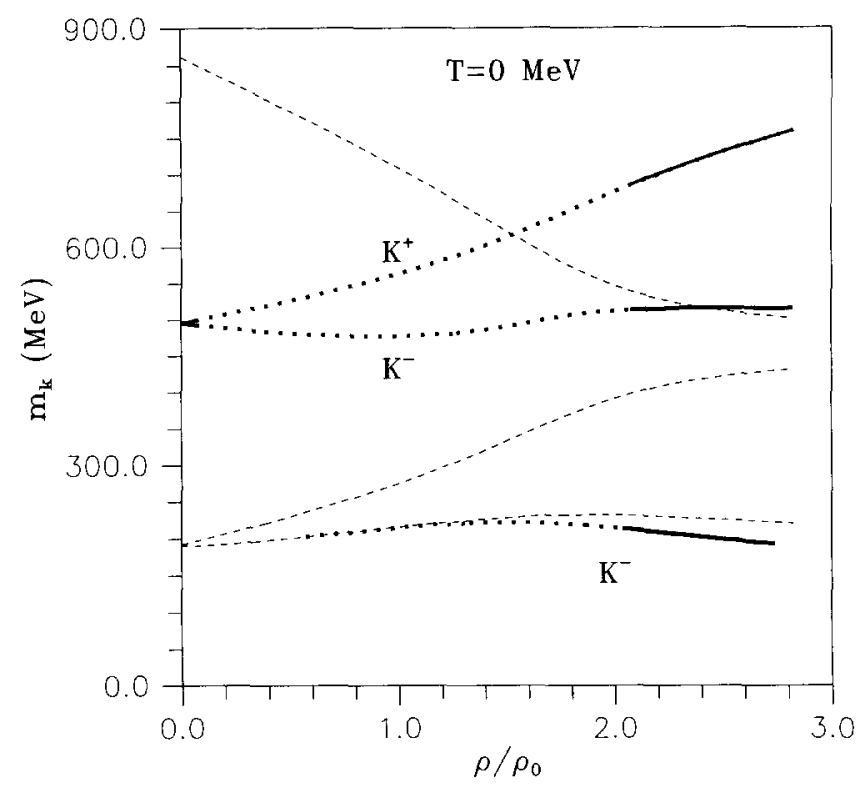

Fig. 2. Kaon masses in symmetric nuclear matter as a function of baryonic density at $T=0 \mathrm{MeV}$, for model 1. The lower curve refers to the $s \bar{u}$ low-lying mode starting at $\rho=0.6 \rho_{0}$. The dotted region coincides with the mixed phase. The two lower dashed curves represent the boundaries of the continuum of the Fermi sea. The upper dashed curve represents the threshold $M_{u}+M_{s}$.

The inclusion of the vector-pseudovector interaction also has important consequences for the kaonic behavior, as can be seen by comparing the results of model I and II. For model I (see Fig. 2), as already reported in [7], we observe a splitting between the masses of $K^{+}$and $K^{-}$excitations of the Dirac sea, the increase in $m_{K^{+}}$being due to Pauli blocking effects. The effect of the Fermi sea particle-hole excitation continuum is strong enough to induce, above certain densities, an increase of the upper $K^{-}$mass and the occurrence of a sub-threshold $K^{-}$-like mode. This low-lying excitation is first embedded in the Fermi sea continuum and, only at densities where the attraction is strong enough $\left(\rho=0.6 \rho_{0}\right)$, it decouples from the continuum. We notice that, since the system is in a mixed phase in the range of densities between $0.1 \rho_{0}$ and $2 \rho_{0}$, the solutions found for the kaon masses (dotted lines of Fig. 2) should be interpreted as average values.

Some comments are in order concerning the low-energy branch, which has a nature different from the upper $\mathrm{K}^{+}, \mathrm{K}^{-}$modes. As a matter of fact, while these modes are excitations of the Dirac sea, the low-energy branch is a particle-hole excitation of the Fermi sea. Branches with a similar origin have been found in other models $[4,11]$ and were interpreted as hyperon-particle-nucleon-hole states. Let us discuss which meaning should be assigned to such a low-energy branch in the present case.

It is well known that the hyperons located below the $\bar{K}-N$ threshold are of particular importance for the $\bar{K}-N$ dynamics. In particular, the $\Lambda(1405)$ seems to play an essential role on the behavior of the in-medium $K^{-}$. In a $\bar{K}-N$ system one can have, besides 


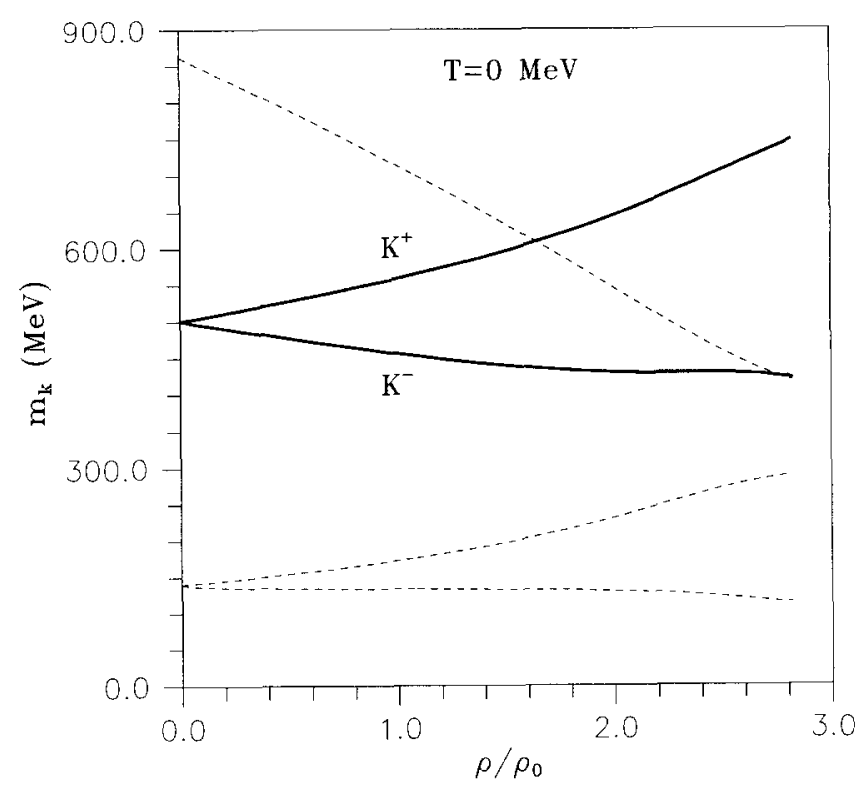

Fig. 3. Kaon masses in symmetric nuclear matter as a function of baryonic density at $T=0 \mathrm{MeV}$, for model II. The dashed curves have a similar meaning to those of Fig. 2 , but with a shifting of $-2 g_{V} \rho_{u}$.

the $A(1405)$ resonance a $A(1405)$-particle-proton-hole state, which is the analogue of Migdal's $\pi_{s}^{+}$mode in a $\pi-N$ system. In NJL type models one does not have bound nucleons and hyperons and an extrapolation from a quark system to a baryon system should be made. Keeping in mind these limitations of the model one can interpret the subthreshold mode (an $s$-particle- $u$-hole excitation) as simulating the effects of a $A$-particle-proton-hole state. Parity analysis leads us to consider this excitation as the analogue of the $A$ (1116)-particle-proton-hole state. The excitation energy of the mode ( $\simeq 200 \mathrm{MeV}$ at $\rho \simeq 0.6 \rho_{0}$ ) also makes this interpretation plausible.

As pointed out in our previous works $[7,8]$, the energy weighted sum rule gives us important information on the character of these two branches: the lower branch, initially weak collective, acquires strength as the density increases and, at a certain density, overtakes the $K^{-}$branch which looses strength, becoming, therefore, less bound.

The results for model II (see Fig. 3) show that the vector-pseudovector interaction term partially cancels some Fermi sea effects: (i) it reduces the repulsion above the Fermi sea continuum, the net effect being a moderate decrease of the mass of the $K^{-}$ Dirac sea excitation; (ii) it reduces the attraction below the Fermi sea continuum, inhibiting therefore the occurrence of the low-lying $s$-particle- $u$-hole mode. This behavior follows qualitatively the same tendency as in [9], where kaon properties in the medium were studied within a chiral effective model and taking into account Pauli blocking, $N N$ correlations and Fermi motion. As in that calculation, the dominant effect we found is Pauli blocking, although, in our case the decrease of the $K^{-}$mass is less pronounced. As a matter of fact, we do not have a bound $s$-particle- $u$-hole state anymore, but we 
still have a narrow continuum of $s$-particle- $u$-hole excitations which acts repulsively in the $K^{-}$mode, as explained above. The $K^{+}$is only slightly affected by the vector interaction.

It is illustrative to look also at the limits of the Fermi sea continuum in both models (see Figs. 2 and 3 ). In model II the continuum is shifted below and narrowed. The shifting is mainly due to a change of $M_{u}=335 \mathrm{MeV}$, in model $\mathrm{I}$, to $M_{u}=369 \mathrm{MeV}$, in model II, which was dictated by the requirement of fitting the parameters to the experimental data. On the other hand, the boundaries of this continuum are now $M_{s}-$ $M_{u}-2 g_{v} \rho_{u}, \mu_{s}-\mu_{u}-2 g_{V} \rho_{u}$.

To the best of our knowledge there is no definitive answer on the way the phase transition occurs in nature [27], especially when asymmetric quark matter is considered, so there is no reason to prefer one of the two models considered here on the basis of experience or phenomenology concerning the phase transition. However, the model II has the advantage of allowing the description of the system in the hadronic phase (in model I this does not happen in the energy range $\left.\left(0.1 \rho_{0}, 2 \rho_{0}\right)\right)$.

\subsection{Combined effects of density and temperature}

From now on we are going to discuss the combined effect of temperature and density on kaonic modes. The interesting thing to be noticed in model $\mathrm{I}$ is that the $s$-particle- $u$ hole collective excitation is only present at zero and very low temperatures. Afterwards the behavior of the kaon modes is qualitatively similar to the observed in model II. In the following we concentrate in the discussions on the results for model II.

At low temperatures the masses of the kaon modes exhibit a behavior identical to the one observed at $T=0 \mathrm{MeV}$ (see Fig. 4). For higher temperatures the behaviour of the kaon masses is still qualitatively similar to the one observed at lower temperatures. However, we can notice that the splitting between $K^{+}$and $K^{-}$decreases with temperature at a given density. The behavior of $K^{ \pm}$with temperature and density is determined by two competing processes. On the one hand, the temperature increases the number of available states to the collective excitations; on the other, the non-strange quark condensate starts increasing with $T$ at a given density. While the first mechanism is dominant for the behavior of $K^{+}$, the behavior of $K^{-}$is a balance between both.

We notice that the dominant effect is the reduction of the splitting between $K^{+}, K^{-}$ masses, which, however, remains still meaningful. For instance, at $T=100 \mathrm{MeV}$ the attractive effect on $K^{+}$is more pronounced but we also observe that, above $\rho \simeq$ $2 \rho_{0}$, the $K^{-}$mass starts to decrease more strongly. At $T=100 \mathrm{MeV} \rho \simeq 2.5 \rho_{0}$ an appreciable splitting, around $200 \mathrm{MeV}$, is found. This means that under the combined effect of temperature and density, the $K^{-}$are still easier to produce in medium, which is compatible with experimental data that indicate an enhancement in $K^{-}$production [15].

Concerning the information from the EWSR, we have checked that in model II the strength exhausted by the collective bound states shows an identically satisfactory behavior, although at high densities the percentage of the total EWSR exhausted by the collective modes is lower, as can be seen from Fig. 5 for $T=100 \mathrm{MeV}$. This is probably 


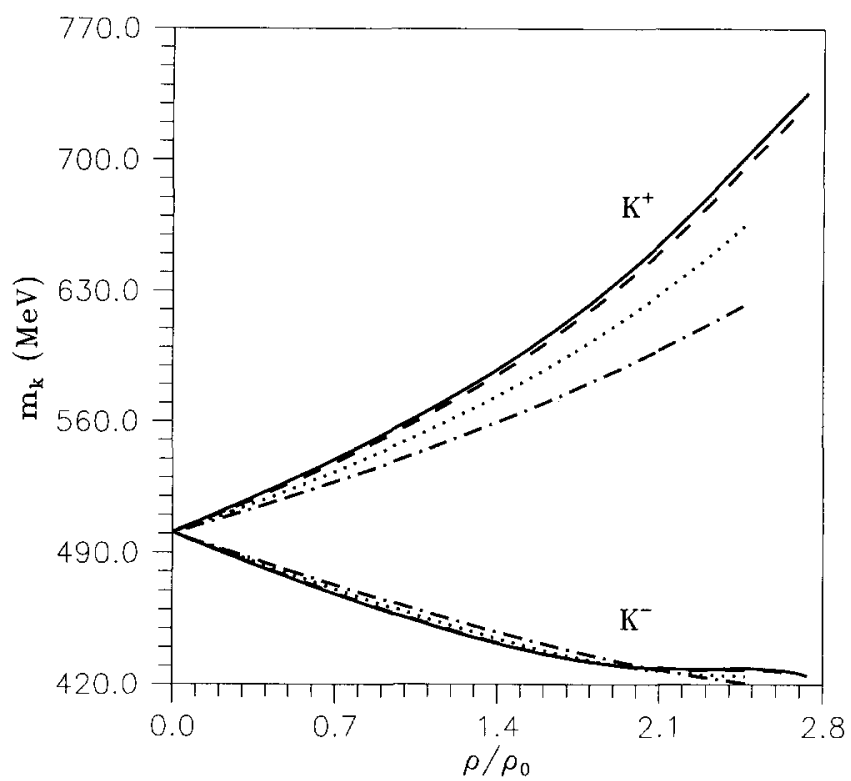

Fig. 4. Kaon masses in symmetric nuclear matter as a function of baryonic density at $T=0 \mathrm{MeV}$ ( $\mathrm{solid}$ curves), $T=20 \mathrm{MeV}$ (dashed curves), $T=50 \mathrm{MeV}$ (dotted curves) and $T=100 \mathrm{MeV}$ (dashed-dotted curves) for model II.

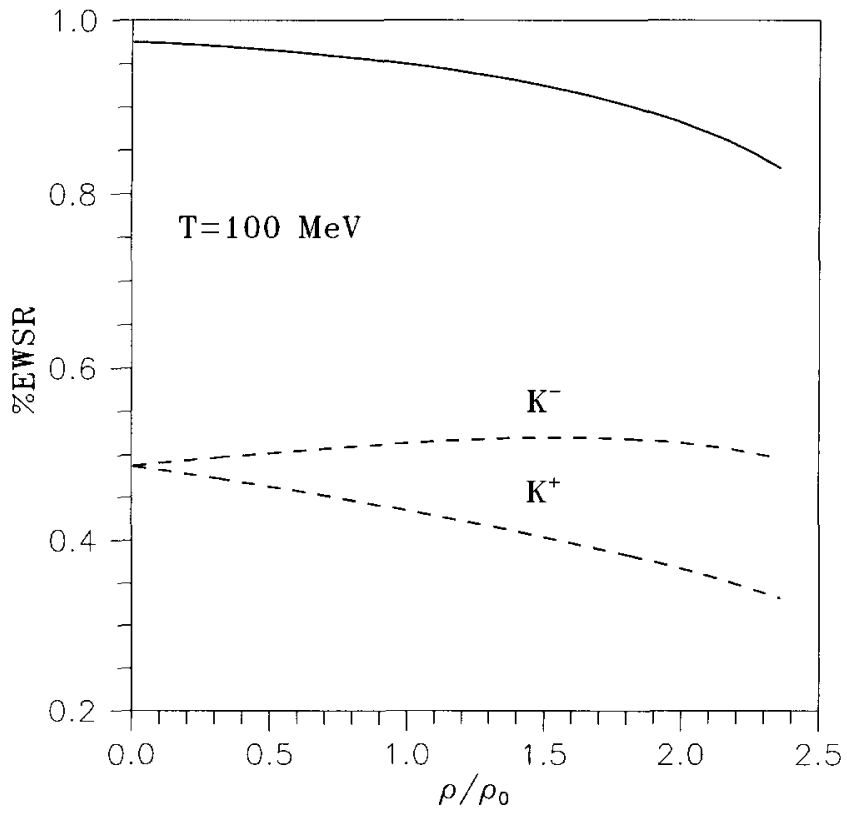

Fig. 5. Percentages of the energy weighted sum rule exhausted by the $K^{ \pm}$bound states as a function of baryonic density at $T=100 \mathrm{MeV}$ for model II. The sum of these percentages is represented by the full line. 
due to the fact that the continuum modes, which are not taken into account in the sum rule, acquire more strength.

\section{Conclusions}

Let us summarize our main results. We have studied properties of the vacuum and of kaons in a flavor-asymmetric medium and at finite temperature, paying special attention to the effects of the Fermi sea, within two SU(3) NJL type models, one without (I) and the other with (II) vector mesons. At zero temperature a first-order phase transition occurs in model I, while in model II a second-order one takes place; the increase of the temperature has the effect of turning out the phase transition to second order in model I. Concerning the behavior of kaons, we conclude that the vector-pseudovector interaction reduces the effects of the Fermi sea. As a consequence, $K^{-}$is a smoothly decreasing function of density and the low energy $s$-particle- $u$-hole excitation of the Fermi sea, present in model I, does not exist as a collective state in model II. A very low temperature regime does not affect noticeably the behavior of kaons. As the temperature increases a moderate reduction of the splitting between the kaon modes is the dominant effect.

\section{Acknowledgements}

We would like to thank $\mathbf{J}$. da Providência for fruitful discussions. We thank the referee for useful comments. This work has been supported in part by projects PRAXIS/PCEX/ C/13/96, CERN/S/FIS/1034/95, ESO PESO/S/PRO/1057/95, PRAXIS/2/2.1/FIS/ 451/94 and PRAXIS/P/FIS/12247/98.

\section{References}

| 1 | F. Karsch, Quark-gluon plasma, ed. R.C. Hwa (World Scientific, Singapore, 1990).

$12 \mid$ W. Cassing and E.L. Bratkovskaya, Phys. Rep. 308 (1999) 65.

13] D.B. Kaplan and A.E. Nelson, Phys. Lett. B 175 (1986) 57; B 192 (1987) 193.

14] H. Yabu, S. Nakamura, F. Myhrer and K. Kubodera, Phys. Lett. B 315 (1993) 17.

$15 \mid$ M. Lutz, A. Steiner and W. Weise, Nucl. Phys. A 574 (1994) 755.

16| J. Schaffner, A. Gal, I.N. Mishustin, H. Stöcker and W. Greiner, Phys. Lett. B 334 (1994) 268.

171 M.C. Ruivo, C.A. de Sousa, Phys. Lett. B 385 (1996) 39.

[81 C.A. de Sousa and M.C. Ruivo, Nucl. Phys. A 625 (1997) 713.

[9] T. Waas, N. Kaiser and W. Weise, Phys. Lett. B 379 ( 1996) 34; T. Waas, M. Rho and W. Weise, Nucl. Phys. A 617 (1997) 449.

[10] G.Q. LI, C.-H. Lee and G.E. Brown, Nucl. Phys. A 625 (1997) 372.

[11] M. Lutz, Phys. Lett. B 426 (1998) 12.

[12] E. Friedmann, A. Gal and C.J. Batty, Nucl. Phys. A 579 (1994) 518.

[13] A.H. Schröter et al., Z. Phys. A 350 (1994) 101.

[14] H. Herrman, FOPI Collaboration, Nucl. Phys. A 610 (1996) 49c.

[15] R. Barth et al., KaoS Collaboration, Phys. Rev. Lett. 78 ( 1997) 4007.

[16] Y. Nambu and G. Jona-Lasinio, Phys. Rev. 122 (1961) 345; 124 (1961) 246. 
[17] M. Jaminon, G. Ripka and P. Stassart, Nucl. Phys. A 504 (1989) 733.

$118]$ M. Asakawa and K. Yazaki, Nucl. Phys. A 504 (1989) 668.

[19] S.P. Klevansky, Rev. Mod. Phys. 64 (1992) 649;

P. Zuang, J. Hüfner and S.P. Klevansky, Nucl. Phys. A 576 (1994) 525.

[20] J. da Providência, M.C. Ruivo and C.A. de Sousa, Phys. Rev. D 36 (1987) 1882;

C.A. de Sousa, Z. Phys. C 43 (1989) 503;

M.C. Ruivo, C.A. de Sousa, B. Hiller and A.H. Blin, Nucl. Phys. A 575 (1994) 460.

121] T. Hatsuda and T. Kunihiro, Phys. Rep. 247 (1994) 221.

[22] M. Jaminon and B. Van den Bossche, Nucl. Phys. A 582 (1995) 517.

[23] J. Cugnon, M. Jaminon and B. Van den Bossche, Nucl. Phys. A 598 (1996) 515.

[24] S. Klimt, M. Lutz and W. Weise, Phys. Lett. B 249 (1990) 386.

[25] V. Bernard and U.G. Meissner, Nucl. Phys. A 489 (1988) 647.

|26] T. Maruyama, K. Tsushima and A. Faessler, Nucl. Phys. A 537 (1992) 303.

[27] H. Müller, Nucl. Phys. A 618 (1997) 349. 